Soumaya Talbi, ${ }^{1}$ Soufiane Arkhamez, ${ }^{1}$ Mustapha Dib, ${ }^{1}$ Mostafa khouili, ${ }^{1}$ Abderrfia Hafid, ${ }^{1}$ Souad Rabi, ${ }^{2}$ Salha hamri ${ }^{3}$.

${ }^{1}$ Laboratoire de Chimie Organique \& Analytique, Equipe COOA, Université Sultan Moulay Slimane, BP 523, 23000 Beni-Mellal, Morocco. Email: soumaya0796@gmail.com

2 Laboratoire de Chimie Organique et Analytique, Université Sultan Moulay Slimane, FST Béni-Mellal, BP 523, 23000 Béni-Mellal, Morocco

${ }^{3}$ Laboratoire de chimie organique, organométallique et valorisation des substances naturelles, Faculté des sciences, université ibn zohr, BP 8106, Dakhla Agadir 80000.

\title{
INTRODUCTION
}

The chemistry of heterocycles has experienced a boom in recent decades. which has resulted in an impressive number of compounds containing in their structure at least one heterocycle. In fact, of the 65 million chemical compounds, more than two thirds contain a heterocyclic system (2009 statistics). Heterocycles are important, not only because of their abundance and extraordinary diversity, but above all because of their usefulness in the biological, medicinal and (vitamins, hormones, antibiotics, etc.), that in the industrial sector, and technological (corrosion inhibitors, dyes, stabilizers, pesticides, herbicides, etc)..

Among the different classes of heterocyclic compounds, mainly nitrogenous structures are present in many natural compounds of plant, animal or synthetic origin. Among these are the pyrazolo-enaminones.

\section{Synthesis of pyrazolo-enaminones}

(1)
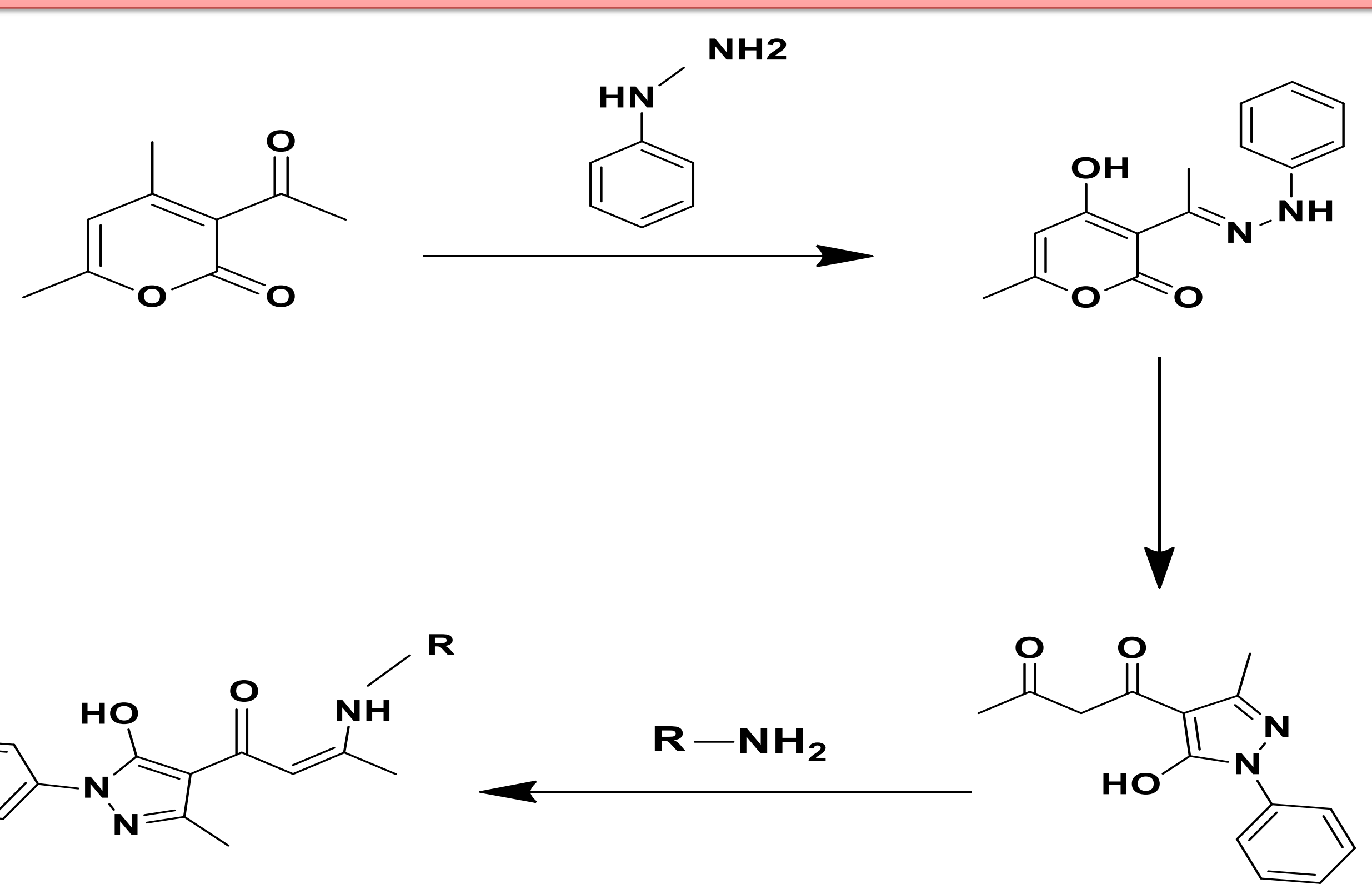

\section{Synthesis of of pyrazolo-enaminones by catalyst $\mathrm{Fe}_{2} \mathrm{O}_{3} \mathrm{Mg} / \mathrm{Al}-\mathrm{LDH}$}

After optimization of the reaction conditions, we prepared a series of pyrazolo-enaminones by condensation of diketo- $N$-phenylpyrazole with different amines using $\mathrm{Fe}_{2} \mathrm{O}_{3}-\mathrm{Mg} / \mathrm{Al}-\mathrm{LDH}$ as catalyst.

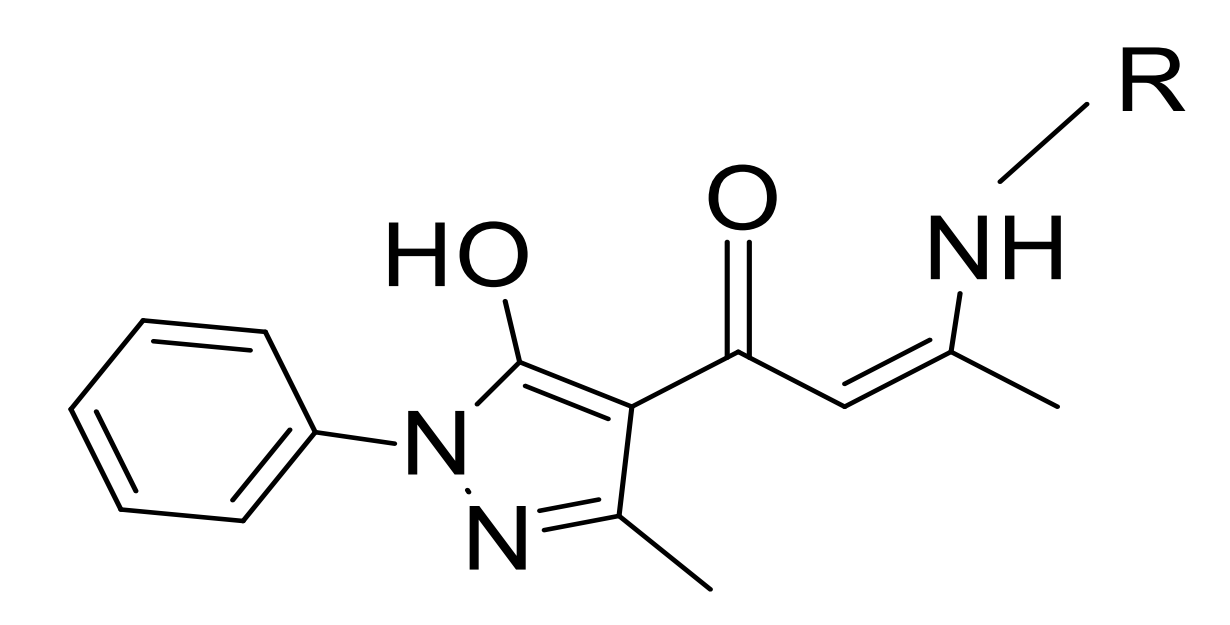

\begin{tabular}{|c|c|c|c|}
\hline Compounds & Rdt \% & Compounds & Rdt \% \\
\hline $\mathrm{R}\left(3-\mathrm{Br}-\mathrm{C}_{6} \mathrm{H}_{5}\right)$ & 89 & $\begin{aligned} & \mathrm{R}\left(4-\mathrm{OH}-2-\mathrm{CH}_{3}-\right. \\
&\left.\mathrm{C}_{6} \mathrm{H}_{5}\right)\end{aligned}$ & 68 \\
\hline $\mathrm{R}\left(\mathrm{C}_{6} \mathrm{H}_{5}\right)$ & 82 & $\mathrm{R}\left(\mathbf{4}-\mathrm{F}-\mathrm{C}_{6} \mathrm{H}_{5}\right)$ & 83 \\
\hline $\begin{array}{l}\mathrm{R}\left(4-\mathrm{OCH}_{3}-2 \mathrm{NO}_{2}-\right. \\
\left.\mathrm{C}_{6} \mathrm{H}_{5}\right)\end{array}$ & 79 & $\mathrm{R}\left(4-\mathrm{OH}-\mathrm{C}_{6} \mathrm{H}_{5}\right)$ & 81 \\
\hline $\begin{array}{l}\mathrm{R}\left(2-\mathrm{CH}_{3}-3 \mathrm{NO}_{2}-\right. \\
\left.\mathrm{C}_{6} \mathrm{H}_{5}\right)\end{array}$ & 89 & $\mathrm{R}\left(\mathbf{2}-\mathbf{N H}_{\mathbf{2}}-\mathrm{C}_{6} \mathbf{H}_{5}\right)$ & 72 \\
\hline $\mathrm{R}\left(4-\mathrm{Cl}-\mathrm{C}_{6} \mathrm{H}_{5}\right)$ & 85 & $\mathrm{R}\left(\mathbf{C}_{3} \mathbf{H}_{3} \mathbf{N S}\right)$ & 84 \\
\hline R 3-OH- $\mathrm{C}_{6} \mathrm{H}_{5}()$ & 78 & $\mathrm{R}\left(\mathrm{C}_{4} \mathrm{H}_{4} \mathrm{~N}_{2}\right)$ & 80 \\
\hline $\mathrm{R}\left(4-\mathrm{NH}_{2} \mathrm{SO}_{2}-\mathrm{C}_{6} \mathrm{H}_{5}\right)$ & 69 & $\mathrm{R}\left(4-\mathrm{OCH}_{3}-\mathrm{C}_{6} \mathrm{H}_{5}\right)$ & 78 \\
\hline
\end{tabular}

\section{References}

(1). Bendaas, A.; Maamar, H. J. Heterocycl. Chem. 1999.36, 1291.

(2) .T. Toraishi, S. Nagasaki, S. Tanaka, Appl. Clay Sci. 22 (2002) 17-23.

(3). Newman SP, Jones W. J. Solid State Chem. 148 (1999) 26 - 40

\section{Attempts synthesis of some pyrazolo-enaminones structures}

Development of a general strategy for obtaining pyrazoloenaminones.
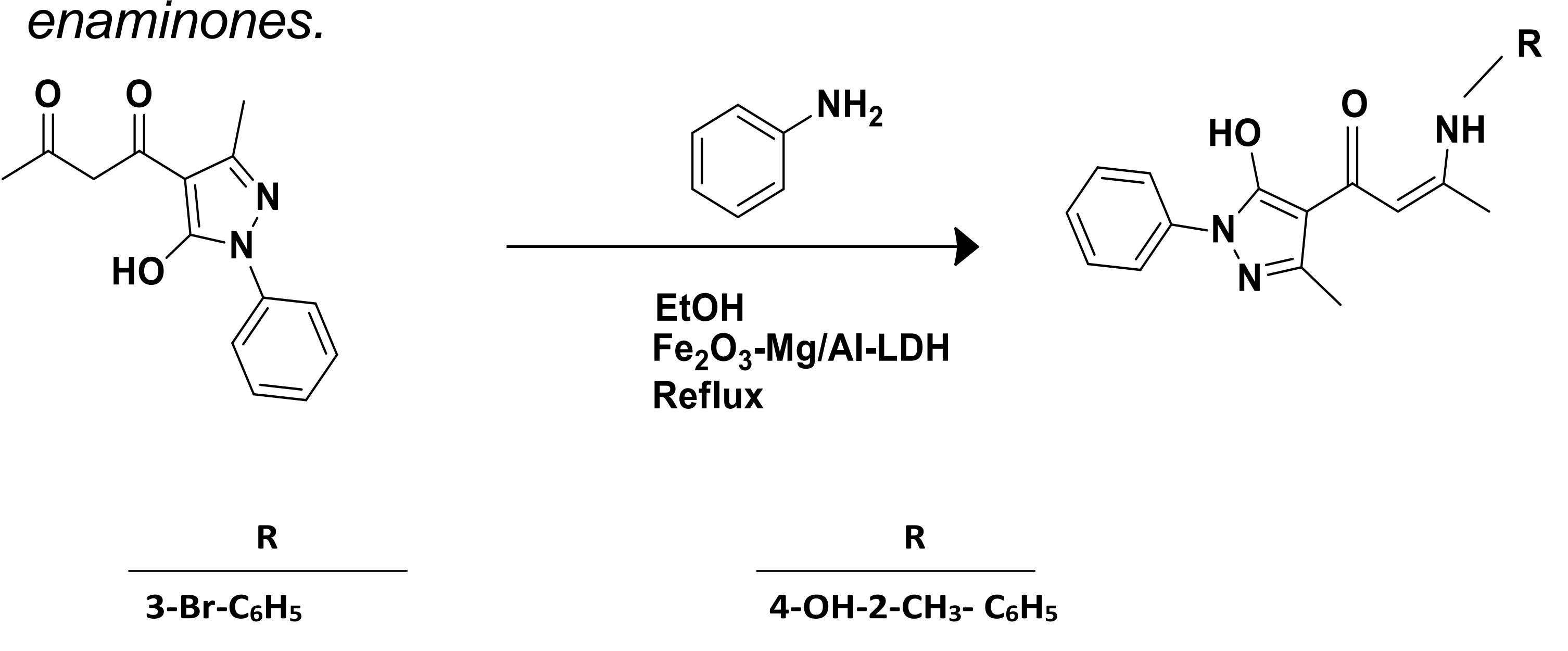

$\mathrm{C}_{6} \mathrm{H}_{5}$

4- $-\mathrm{OCH}_{3}-2 \mathrm{NO}_{2}-\mathrm{C}_{6} \mathrm{H}_{5}$

$2-\mathrm{CH}_{3}-3 \mathrm{NO}_{2}-\mathrm{C}_{6} \mathrm{H}_{5}$

4- $\mathrm{Cl}-\mathrm{C}_{6} \mathrm{H}_{5}$

3-OH- $\mathrm{C}_{6} \mathrm{H}_{5}$

4- $\mathrm{NH}_{2} \mathrm{SO}_{2}-\mathrm{C}_{6} \mathrm{H}_{5}$

$\frac{1}{\text { 4-OH-2- }-\mathrm{CH}_{3}-\mathrm{C}_{6} \mathrm{H}_{5}}$
4-F- $\mathrm{C}_{6} \mathrm{H}_{5}$
4-OH- $\mathrm{C}_{6} \mathrm{H}_{5}$
2- $\mathrm{NH}_{2}-\mathrm{C}_{6} \mathrm{H}_{5}$
$\mathrm{C}_{3} \mathrm{H}_{3} \mathrm{NS}$
$\mathrm{C}_{4} \mathrm{H}_{4} \mathrm{~N}_{2}$
$4-\mathrm{OCH}_{3}-\mathrm{C}_{6} \mathrm{H}_{5}$

(2), (3)

\section{conclusion}

A series of enaminones was synthesized with good yields under simple conditions.

We expect that these substrates will become useful building blocks for the preparation of other heterocycles. 